\title{
Clinical and hematologic manifestations in patients with Diamond Blackfan anemia in Korea
}

\author{
Soon-Ki Kim ${ }^{1}$, Hyo-Seop $\mathrm{Ahn}^{2}$, Hee-Jo Back ${ }^{3}$, Bin Cho ${ }^{4}$, Eun-Jin Choi ${ }^{5}$, Nak-Gyun Chung ${ }^{4}$, \\ Pyoung-Han Hwang ${ }^{6}$, Dae-Chul Jeoung ${ }^{4}$, Hyung-Jin Kang ${ }^{2}$, Hyery Kim² ${ }^{2}$ Kyung-Nam Ko ${ }^{7}$, \\ Hong-Hoe Koo ${ }^{8}$, Hoon Kook ${ }^{3}$, Kwang-Chul Lee ${ }^{9}$, Ho-Joon Lim ${ }^{7}$, Young-Tak Lim ${ }^{10}$, Chuhl-Joo Lyu ${ }^{11}$, \\ Jun-Eun Park ${ }^{12}$, Kyung-Duk Park ${ }^{2}$, Sang-Kyu Park ${ }^{7}$, Kyung-Ha Ryu ${ }^{13}$, Jong-Jin Seo ${ }^{7}$, Hee-Young Shin ${ }^{2}$, \\ Ki-Woong Sung ${ }^{8}$, Eun Sun Yoo ${ }^{13}$ \\ Department of Pediatrics, College of Medicine, ${ }^{1}$ Inha University, ${ }^{2}$ Seoul National University, ${ }^{3}$ Chonnnam National University, \\ ${ }^{4}$ Catholic University, ${ }^{5}$ Daegu Catholic University, ${ }^{6}$ Chonbuk National University, ${ }^{7}$ University of Ulsan, ${ }^{8}$ Sungkyunkwan University, \\ ${ }^{9}$ Korea University, ${ }^{10}$ Pusan National University, ${ }^{11}$ Yonsei University, ${ }^{12}$ Ajou University, ${ }^{13}$ Ewha Womans University, Korea
}

p-ISSN 1738-7949 / e-ISSN 2092-9129

http://dx.doi.org/10.5045/kjh.2012.47.2.131

Korean J Hematol 2012;47:131-5.

Received on March 3, 2012

Revised on May 16, 2012

Accepted on May 18, 2012

\section{Background}

Diamond Blackfan anemia (DBA), characterized by impaired red cell production, is a rare condition that is usually symptomatic in early infancy. The purpose of this study was to assess nationwide experiences of DBA encountered over a period of 20 years.

\section{Methods}

The medical records of 56 patients diagnosed with DBA were retrospectively reviewed from November 1984 to July 2010. Fifteen institutions, including 13 university hospitals, participated in this study.

Results

The male-to-female ratio of patients with DBA was 1.67:1. The median age of diagnosis was 4 months, and $74.1 \%$ were diagnosed before 1 year of age. From 2000 to 2009, annual incidence was 6.6 cases per million. Excluding growth retardation, 38.2\% showed congenital defects: thumb deformities, ptosis, coarctation of aorta, ventricular septal defect, strabismus, etc. The mean hemoglobin concentration was $5.1 \pm 1.9 \mathrm{~g} / \mathrm{dL}$, mean corpuscular volume was $93.4 \pm 11.6 \mathrm{fL}$, and mean number of reticulocytes was $19,700 / \mathrm{mm}^{3}$. The mean cellularity of bone marrow was $75 \%$, with myeloid:erythroid ratio of $20.4: 1$. After remission, $48.9 \%$ of patients did not need further steroids. Five patients with DBA who received hematopoietic transplantation have survived. Cancer developed in 2 cases (3.6\%).

\section{Conclusion}

The incidence of DBA is similar to data already published, but our study had a male predilection. Although all patients responded to initial treatment with steroids, about half needed further steroids after remission. It is necessary to collect further data, including information regarding management pathways, from nationwide DBA registries, along with data on molecular analyses.

Key Words Diamond Blackfan anemia, Anemia, Congenital defects

\section{INTRODUCTION}

Diamond Blackfan anemia (DBA; OMIM 205900), characterized by impaired red cell production, is a rare condition that is usually symptomatic in early infancy. DBA was first recognized as a distinct clinical entity in 1938 [1]. The diag- nostic criteria for DBA that were developed in 1976 have remained the accepted standard [2] and include anemia, usually presenting prior to the first birthday, with near normal neutrophil and/or platelet counts, reticulocytopenia, macrocytosis, and normal marrow cellularity with a paucity of red cell precursors. These criteria comprise "classical" DBA. The disorder results from a cellular defect, in which erythroid

This is an Open Access article distributed under the terms of the Creative Commons Attribution Non-Commercial License (http://creativecommons.org/licenses/by-nc/3.0) which permits unrestricted non-commercial use, distribution, and reproduction in any medium, provided the original work is properly cited. 
progenitors and precursors are highly sensitive to death by apoptosis, leading to erythropoietic failure [3-5].

DBA came into the scientific spotlight after the identification of RPS19, the gene encoding ribosomal protein S19 [6]. Since then, many genes that encode large (RPL) and small (RPS) ribosomal subunit proteins have been found to be mutated in DBA patients, such as RPL5, RPL11, RPL35A, RPS7, RPS10, RPS17, RPS24, and RPS26 [7-10].

Although the first case of DBA was reported in 1974 in Korea [11], there is relatively limited data regarding DBA. Recently, direct sequencing analysis of the RPS19 gene was performed with both cDNA and genomic DNA extracted from peripheral blood, and a c.3G $>A$ point mutation in exon 2 resulting in a p.Met1Ile substitution was identified in a 3-month-old infant [12].

In this report, we reviewed the clinical characteristics of 56 patients with DBA from a 20 -year birth cohort in Korea to provide further information for molecular analyses.

\section{MATERIALS AND METHODS}

The medical records of 56 patients diagnosed with DBA were retrospectively reviewed from November 1984 to July 2010. Thirteen university hospitals participated in this study. Diagnostic and supporting criteria for the diagnosis of DBA are as follows: normochromic, often macrocytic anemia, reticulocytopenia, and a low number or lack of erythroid precursors in the bone marrow. In only two patients, diagnoses

Table 1. Clinical and laboratory findings at diagnosis in 56 patients with Diamond Blackfan anemia.

\begin{tabular}{lcc}
\hline Clinical characteristics & $\mathrm{N}$ & $\%$ \\
\hline $\begin{array}{l}\text { Gender } \\
\text { Male }\end{array}$ & 35 & 62.5 \\
$\quad$ Female & 21 & 37.5 \\
$\begin{array}{l}\text { Family history } \\
\text { Yes }\end{array}$ & 3 & 5.4 \\
Age at diagnosis & & \\
$\quad<6$ mo & 31 & 57.4 \\
$\quad<12$ mo & 9 & 16.7 \\
$\quad>12$ mo & 14 & 25.9 \\
Unknown & 2 & 3.6 \\
\hline Laboratory findings & Mean $\pm \mathrm{SD}$ & Range \\
\hline Red blood cells, $10^{6} / \mathrm{mm}^{3}$ & $1.88 \pm 0.93$ & $0.49-4.7$ \\
Hemoglobin, g/dL & $5.1 \pm 2.1$ & $1.5-9.8$ \\
MCV, fL & $93.4 \pm 11.6$ & $74.0-116.6$ \\
MCH, pg & $31.1 \pm 4.0$ & $23.4-40.3$ \\
HbF, $\%$ & $7.7 \pm 11.3$ & $0-49.9$ \\
Adenosine deaminase, $\mathrm{U} / \mathrm{L}$ & $21.1 \pm 13.7$ & $3.5-48.5$ \\
\hline Bone marrow & & \\
\hline Cellularity & & \\
M:E ratio & $75.3 \pm 20.4$ & $30-100$ \\
\hline
\end{tabular}

Abbreviations: $\mathrm{MCV}$, mean corpuscular volume; $\mathrm{MCH}$, mean corpuscular hemoglobin; $\mathrm{HbF}$, fetal hemoglobin; M:E ratio, myeloid:erythroid ratio. were confirmed by mutation analyses [12]. Clinical information, including records on birth history, age of onset and diagnosis, family history, height and weight, malformations, hematologic data, response to therapeutic procedures, and prognosis, was obtained via a standardized questionnaire. Remission was defined as a stable, physiologically acceptable hemoglobin $(\mathrm{Hb})$ level maintained for at least 6 months independent of corticosteroids, transfusions, or other therapy.

Means and standard deviations were used in the statistical analysis. Statistical significance was defined as $P<0.05$.

\section{RESULTS}

The male to female ratio of patients was 1.67:1 (35 males, 21 females) as shown in Table 1 . Among the patients diagnosed after 2000, the male-to-female ratio was 1.62:1 (21 males, 13 females). Only 3 cases (5.4\%) revealed a family history. However, the genetic study was not done in the patients and family members.

The median age of diagnosis was 4 months, and the range was birth to 52 months of age. The proportion of patients diagnosed before 1 year was $74.1 \%$ (40/54), excluding 2 patients without age documents at diagnosis. From 2000 to 2009, 33 cases with DBA were registered, yielding an incidence of 6.6 cases per million, based on the Korean annual birth rate of 500,000 . The mean $\mathrm{Hb}$ concentration was $5.0 \pm 1.8$ $\mathrm{g} / \mathrm{dL}$ (range, 1.5-9.8 g/dL), and the median was $5.2 \mathrm{~g} / \mathrm{dL}$.

Table 2. Congenital anomalies observed in 56 patients with Diamond Blackfan anemia.

\begin{tabular}{lll}
\hline & & $\mathrm{N}$ \\
\hline Craniofacial & Cleft palate & 2 \\
Ophthalmologic & Ptosis & 5 \\
& Strabismus & 3 \\
& Entropion & 1 \\
Neck & Webbed neck & 1 \\
& Klippel-Feil syndrome & 1 \\
& Short neck & 1 \\
Thumbs & Thumb deformities & 8 \\
Cardiac & VSD & 4 \\
& ASD & 1 \\
& Complex cardiac anomalies & 1 \\
& Coarctation of aorta & 5 \\
Other musculoskeletal & Mitral valve prolapse & 2 \\
& Growth retardation & 4 \\
Neuromotor & Failure to thrive & 1 \\
Total & Hearing loss & 1 \\
& & 41 \\
\hline
\end{tabular}

Abbreviations: VSD, ventricular septal defect; ASD, atrial septal defect. 
The mean value of the mean corpuscular volume (MCV) was $93.4 \pm 11.6 \mathrm{fL}$ and mean fetal hemoglobin $(\mathrm{HbF})$ level was $7.7 \pm 11.3 \%$. The mean number of corrected reticulocytes was markedly lower than normal $\left(19,700 / \mathrm{mm}^{3}\right)$. The mean adenosine deaminase level was higher than normal, at $21.3 \pm 14.4 \mathrm{U} / \mathrm{L}$. The mean cellularity of the bone marrow was $75 \%$, and the myeloid:erythroid (M:E) ratio was 20.4:1 (range, 1.9:1 to 100:1). Among 28 patients with chromosomal analysis data, 26 showed a normal pattern in routine cytogenetic analysis. Direct sequencing analysis of the RPS19 gene was performed in 2 patients, who had the substitutions c.357-2A > G (RPS19) and c.3G > A (Met1Ile), respectively.

Excluding growth retardation, 21 cases $(38.2 \%)$ showed congenital defects: thumb deformities (8 cases), ptosis (5), coarctation of aorta (5), ventricular septal defect (4), strabismus (3), cleft palate (2), and mitral valve prolapse (2). More than one anomaly was found in $21.2 \%$ of individuals (Table 2).

Thirty-two patients had a history of transfusion after the initial correction of $\mathrm{Hb}$ at diagnosis. All patients initially received corticosteroids, and $83.9 \%$ (47/56) responded with an increase in $\mathrm{Hb}$. In $48.9 \%$ (23/47) of patients, steroids were eventually stopped with continued maintenance of adequate hemoglobin levels. The mean duration of steroid use was 7 years (maximum, 22 yrs). Complications due to steroid therapy were reported in 31 cases, including growth retardation (13), moon face (6), hirsutism (3), impaired glucose tolerance (3), and diabetes mellitus (2) (Table 3). Among the 28 patients who received steroids, 9 patients were on a well-controlled regimen, with a daily dose of $5 \mathrm{mg}$ or less.

Iron chelation therapy was attempted in 6 patients with high serum ferritin levels (usually more than $1,000 \mathrm{ng} / \mathrm{mL}$ ): deferoxamine (Desferal) intravenously in 3 cases, and more recently, deferasirox (Exjade) in 4 cases.

Five patients underwent hematopoietic stem cell transplantation (HSCT) at $81 \pm 46$ months (range, $22-150$ months).

Table 3. Side effects of steroid treatment in patients with Diamond Blackfan anemia.

\begin{tabular}{llr}
\hline & & $\mathrm{N}$ \\
\hline Cosmetic & Hirsutism & 3 \\
& Moon face & 6 \\
& Weight gain & 1 \\
Behavioral & Depression & 1 \\
Endocrine & Impaired glucose tolerance & 3 \\
& Diabetes mellitus & 2 \\
Growth & Menstrual irregularities & 1 \\
Immunosuppression & Impaired growth velocity & 13 \\
Total & Candida & 1 \\
\hline
\end{tabular}

Transplantation sources were bone marrow from related donors (3 patients), bone marrow from unrelated donors (1 patient), and peripheral blood from unrelated donor (1 patient). Four recipients underwent conditioning without irradiation. Graft-versus-host disease prophylaxis included methotrexate (3 patients) and antithymocyte globulin (3 patients). All 5 patients have survived. Only one patient was lost to follow-up at the well-being state.

In the median follow-up of 8 years, there were 2 cases (3.6\%) of malignancy: diffuse large cell lymphoma after 8 months and osteosarcoma after 13 years of DBA. The latter died due to lung metastasis and pulmonary hypertension.

\section{DISCUSSION}

DBA is characterized by macrocytic anemia, reticulocytopenia, and normal bone marrow cellularity with a paucity of erythroid precursors. DBA is caused by a defect in which erythroid progenitors and precursors are highly sensitive to death by apoptosis, leading to erythropoietic failure [4, 5, 13].

As in this study, routine cytogenetic analysis of patients with DBA is normal. However, a balanced reciprocal translocation $\mathrm{t}(\mathrm{X} ; 19)$ was discovered in a DBA patient [6], revealing the first DBA gene ( $D B A 1)$ that encodes a ribosomal protein S19 (RPS19) that is essential for the maturation of the $40 \mathrm{~S}$ ribosomal subunit [14]. The identification of subsequent DBA genes has established DBA as a ribosomal disorder, because the affected gene encodes ribosomal proteins [8]. RPS19 protein haploinsufficiency is not sufficient to produce normal function. Recently, de novo mutations have been identified in ribosomal proteins RPS24 [8], RPS17, RPL5, RPL11, and RPL35a. To date, approximately 50\% of DBA patients have a single mutation in a gene encoding a ribosomal protein. These findings implicate DBA as a disorder of ribosome biogenesis and/or function.

The diagnostic criteria for "classical" DBA, presented in 1976 [2], include the followings: anemia, usually presenting prior to the first birthday, with near normal, but variable, neutrophil and/or platelet counts; reticulocytopenia; macrocytosis; and normal marrow cellularity with a paucity of red cell precursors. "Non-classical" DBA is characterized by presentation at an age $>1$ year, with congenital anomalies, no anemia, a mild hematological phenotype (macrocytosis only), or the presence of a mutation shared by affected family members.

Although all immediate family members should be evaluated with a thorough relevant history, and a complete blood count, including red cell indices, erythrocyte adenosine deaminase (eADA) activity, and $\mathrm{HbF}$, these were not completely performed in our study. This may be the reason why only 3 cases in our study revealed a family history. Generally, 40-45\% of DBA cases are familial with autosomal dominant inheritance [15], and the remainder is sporadic or familial with seemingly different patterns of inheritance.

In a study of $80 \mathrm{DBA}$ patients in the United Kingdom 
[16], 35\% of classical DBA patients were found to have one or more unequivocal congenital anomalies, not including growth retardation. A similar proportion has been described in the French, Italian, and North American registries (40\%, $46 \%$, and $47 \%$, respectively) $[3,17,18]$. More than one anomaly was found in up to $25 \%$ of individuals. In this study, 21 cases (38.2\%) showed congenital defects, with more than one anomaly in $21.2 \%$. Renal anomalies have not been seen. However, in the North American Diamond Blackfan Anemia Registry (DBAR), where the majority of patients undergo formal genitourinary and cardiac imaging, the prevalence is $19 \%$.

The incidence of DBA is estimated to be between 1 in 100,000 and 1 in 200,000 without ethnic predilection, and both sexes are reported to be equally affected [3, 16-19]. In our study, the prevalence of DBA was similar to these statistics (6.6 cases per million), but with a male predilection (M:F=1.67:1).

The reported proportion of cancer is $4 \%(29 / 700)$ in patients with DBA, which is higher than the expected $0.5 \%$ for a cohort less than 30 years of age [20, 21]. The median age for cancer in these reports was 15 years, much younger than the median of 68 years in the general population [22]. The crude frequency reported from Boston Children's Hospital was $6.6 \%$ [23], the French series 3.8\% [18], and the DBAR $1.9 \%(8 / 420)$ [3]. In this study, 2 cases $(3.6 \%)$ with DBA developed cancer: osteogenic sarcoma and diffuse large cell lymphoma, respectively.

Corticosteroids remain the mainstay of DBA treatment. The therapeutic mechanism of corticosteroids in DBA may involve increased sensitivity to erythropoietin (Epo) $[4,24]$. Remission occurred in $48.9 \%$. The initial recommended starting dose was $2 \mathrm{mg} / \mathrm{kg}$ prednisone or the glucocorticoid equivalent. Most patients are controlled with the recommended maximum maintenance dose $(\leq 1 \mathrm{mg} / \mathrm{kg}$ every other day or $\leq 0.5 \mathrm{mg} / \mathrm{kg}$ daily). Seventy percent of those who achieved remission did so within the first decade of life.

Complications due to steroid therapy were noted in $20 \%$ of patients on long-term steroid therapy [18], and $22 \%$ of those in the DBAR [3]. In our study, growth retardation was most common, followed by moon face, hirsutism, impaired glucose tolerance, and diabetes mellitus. Growth hormone therapy has been used successfully to increase growth velocity [25]. In our study, 1 patient who had been diagnosed with growth hormone deficiency showed good response to recombinant growth hormone therapy for 1.5 yrs (height increased from less than the 3rd percentile to the 10th percentile), without bone age advancement.

Addition of iron-chelating therapy is recommended in transfusion-dependent patients, because prolonged iron acquisition from transfusion can cause a potentially fatal iron overload in the liver, heart, or pancreas. Iron chelation was initiated when a ferritin level was $1,000 \mathrm{ng} / \mathrm{mL}$ or more instead of the hepatic iron concentration reaching 6-7 mg/g dry weight. Chelation by subcutaneous deferoxamine (Desferal, Novartis) was switched in 2007 to oral deferasirox (ICL670, Exjade, Novartis), which is now approved in Korea as the first-line therapy for transfusional iron overload [26].

Hematopoietic stem cell transplantation (HSCT) is the curative treatment for DBA [3, 18, 27, 28]. According to the DBAR [3], where the major indication for HSCT was transfusion dependence, the bone marrow was the most common source of stem cells. The survival rate for allogeneic sibling versus alternative donor transplant was $72.7 \%$ vs. $17.1 \%$ at $>5$ years following SCT. The survival for patients $<10$ years of age who underwent transplantation from human leukocyte antigen (HLA)-matched allogeneic siblings was $92.3 \%$ [19]. More recently, successful related umbilical cord blood and unrelated donor transplants have been reported to the DBAR, but it is too early for a meaningful analysis of the data.

In conclusion, the incidence of DBA is similar to data already published, but there was a male predilection in our study. Although all patients responded to initial treatment with steroids, about half needed further steroids after remission. It is necessary to collect further data, including information on management pathways, from nationwide DBA registries, in addition to molecular analyses. Since DBA patients need lifetime care, they should be managed by a multidisciplinary team involving pediatric and adult hematologists, internists, and subspecialists.

\section{REFERENCES}

1. Diamond LK, Blackfan KD. Hypoplastic anemia. Am J Dis Child 1938;56:464-7.

2. Diamond LK, Wang WC, Alter BP. Congenital hypoplastic anemia. Adv Pediatr 1976;22:349-78.

3. Lipton JM, Atsidaftos E, Zyskind I, Vlachos A. Improving clinical care and elucidating the pathophysiology of Diamond Blackfan anemia: an update from the Diamond Blackfan Anemia Registry. Pediatr Blood Cancer 2006;46:558-64.

4. Ohene-Abuakwa Y, Orfali KA, Marius C, Ball SE. Two-phase culture in Diamond Blackfan anemia: localization of erythroid defect. Blood 2005;105:838-46.

5. Miyake K, Utsugisawa T, Flygare J, et al. Ribosomal protein S19 deficiency leads to reduced proliferation and increased apoptosis but does not affect terminal erythroid differentiation in a cell line model of Diamond-Blackfan anemia. Stem Cells 2008;26:323-9.

6. Draptchinskaia N, Gustavsson P, Andersson B, et al. The gene encoding ribosomal protein S19 is mutated in Diamond-Blackfan anaemia. Nat Genet 1999;21:169-75.

7. Doherty L, Sheen MR, Vlachos A, et al. Ribosomal protein genes RPS10 and RPS26 are commonly mutated in Diamond-Blackfan anemia. Am J Hum Genet 2010;86:222-8.

8. Gazda HT, Grabowska A, Merida-Long LB, et al. Ribosomal protein S24 gene is mutated in Diamond-Blackfan anemia. Am J Hum Genet 2006;79:1110-8.

9. Farrar JE, Nater M, Caywood E, et al. Abnormalities of the large ribosomal subunit protein, Rpl35a, in Diamond-Blackfan anemia. Blood 2008;112:1582-92.

10. Konno Y, Toki T, Tandai S, et al. Mutations in the ribosomal protein genes in Japanese patients with Diamond-Blackfan 
anemia. Haematologica 2010;95:1293-9.

11. Park SW, Shin SM, Shin A, Ko KW, Hong CY. Congenital hypoplastic anemia (Blackfan-Diamond Syndrome). Korean J Hematol 1974;9:19-25.

12. Chae H, Park J, Kim M, et al. Diamond-Blackfan anemia confirmed by RPS19 gene mutation analysis: a case study and literature review of Korean patients. Korean J Lab Med 2010;30:249-54.

13. Lipton JM, Ellis SR. Diamond-Blackfan anemia: diagnosis, treatment, and molecular pathogenesis. Hematol Oncol Clin North Am 2009;23:261-82.

14. Flygare J, Aspesi A, Bailey JC, et al. Human RPS19, the gene mutated in Diamond-Blackfan anemia, encodes a ribosomal protein required for the maturation of $40 \mathrm{~S}$ ribosomal subunits. Blood 2007;109:980-6.

15. Orfali KA, Ohene-Abuakwa Y, Ball SE. Diamond Blackfan anaemia in the UK: clinical and genetic heterogeneity. Br J Haematol 2004;125:243-52.

16. Ball SE, McGuckin CP, Jenkins G, Gordon-Smith EC. DiamondBlackfan anaemia in the U.K.: analysis of 80 cases from a 20-year birth cohort. Br J Haematol 1996;94:645-53.

17. Ramenghi U, Garelli E, Valtolina S, et al. Diamond-Blackfan anaemia in the Italian population. Br J Haematol 1999;104:841-8.

18. Willig TN, Niemeyer CM, Leblanc T, et al. Identification of new prognosis factors from the clinical and epidemiologic analysis of a registry of 229 Diamond-Blackfan anemia patients. DBA group of Société d'Hematologie et d'Immunologie Pédiatrique (SHIP), Gesellshaft für Pädiatrische Onkologie und Hämatologie (GPOH), and the European Society for Pediatric Hematology and Immunology (ESPHI). Pediatr Res 1999;46:553-61.

19. Ohga S, Mugishima H, Ohara A, et al. Diamond-Blackfan anemia in Japan: clinical outcomes of prednisolone therapy and hematopoietic stem cell transplantation. Int J Hematol 2004;79:22-30.
20. Alter BP, Giri N, Savage SA, et al. Malignancies and survival patterns in the National Cancer Institute inherited bone marrow failure syndromes cohort study. Br J Haematol 2010;150:179-88.

21. Yaris N, Erduran E, Cobanoglu U. Hodgkin lymphoma in a child with Diamond Blackfan anemia. J Pediatr Hematol Oncol 2006;28:234-6.

22. Ries LAG, Eisner MP, Kosary CL, et al. SEER Cancer Statistics Review, 1973-1998. Bethesda, MD: National Cancer Institute. (Accessed March 22, 2012, at http://seer.cancer.gov/csr/1973_ 1998.)

23. Janov AJ, Leong T, Nathan DG, Guinan EC. Diamond-Blackfan anemia. Natural history and sequelae of treatment. Medicine (Baltimore) 1996;75:77-8.

24. Ebert BL, Lee MM, Pretz JL, et al. An RNA interference model of RPS19 deficiency in Diamond-Blackfan anemia recapitulates defective hematopoiesis and rescue by dexamethasone: identification of dexamethasone-responsive genes by microarray. Blood 2005;105:4620-6.

25. Scott EG, Haider A, Hord J. Growth hormone therapy for short stature in Diamond Blackfan anemia. Pediatr Blood Cancer 2004;43:542-4.

26. Min JW, Hwang SJ, Lim YJ, Lee YH. Acute tubulointerstitial nephritis induced by deferasirox following hematopoietic stem cell transplantation for severe aplastic anemia. Korean J Hematol 2008;43:258-62.

27. Mugishima $\mathrm{H}$, Ohga $\mathrm{S}$, Ohara A, et al. Hematopoietic stem cell transplantation for Diamond-Blackfan anemia: a report from the Aplastic Anemia Committee of the Japanese Society of Pediatric Hematology. Pediatr Transplant 2007;11:601-7.

28. Vlachos A, Muir E. How I treat Diamond-Blackfan anemia. Blood 2010;116:3715-23. 